\title{
Global, collisional model of high-energy photoelectrons
}

\author{
G. V. Khazanov, T. E. Moore, and M. W. Liemohn \\ Space Sciences Laboratory, NASA/MSFC, Huntsville, Alabama \\ V. K. Jordanova \\ Space Physics Research Laboratory, University of Michigan, Ann Arbor \\ M.-C. Fok \\ USRA/ES83, Space Sciences Laboratory, NASA/MSFC, Huntsville, Alabama
}

\begin{abstract}
A previously-developed colissional, interhemispheric flux tube model for photoelectrons (PE) [Khazanov et $a l ., 1994]$ has been extended to three dimensions by including transport due to $\vec{E} \times \vec{B}$ and magnetic gradient-curvature drifts. Using this model, initial calculations of the high-energy ( $\geq 50$ eV) PE distribution as a function of time, energy, pitch angle, and spatial location in the equatorial plane, are reported for conditions of low geomagnetic activity. To explore both the dynamic and steady behaviors of the model, the simulation starts with the abrupt onset of photoelectron excitation, and is followed to steady state conditions. The results illustrate several features of the interaction of photoelectrons with typical magnetospheric plasmas and fields, including collisional diffusion of photoelectons in pitch angle with flux tube filling, diurnal intensity and pitch angle asymmetries introduced by directional sunlight, and energization of the photoelectron distribution in the evening sector. Cross-field drift is shown to have a long time scale, taking 12 to 24 hours to reach a steady state distribution. Future applications of the model are briefly outlined.
\end{abstract}

\section{Introduction}

An important aspect of photoelectrons (PE) is their transport through the plasmasphere. The PE escaping from the ionosphere experience small-angle scattering when traveling through the plasmasphere as a result of the Coulomb interaction with the thermal plasma. Due to this scattering, some of the PE are scattered to the outside of the loss cone and undergo magnetic reflection, i.e. they become trapped (for a comprehensive review of this problem see Khazanov et. al. [19931995]).

Among the numerous papers devoted to PE transport between the magnetoconjugate regions of the ionosphere, no results have yet been published which describe the evolution and formation of PE fluxes under nonstationary conditions on a global scale. The previous models just picked a single field tube, or part of one, and considered the PE transport problem only inside this restricted region. However, PE calculations are an important part of global modeling of the plasma distribution in the inner magnetosphere. The authors believe that

\section{Copyright 1996 by the American Geophysical Union.}

the investigations of non-steady-state PE transport on a global scale is the logical next step on the path to developing a self-consistent model of the thermal plasma in the inner magnetosphere and can form the basis of a numerical space weather prediction scheme. Such a study also has particular relevance to the goals of the IMI (Inner Magnetosphere Imaging) mission currently being developed at the Marshall Space Flight Center. The temporal evolution and spatial distribution of plasma parameters in the inner magnetosphere and the variation in these quantities with changing solar and magnetic activity are critical factors in setting the sensitivity ranges and spatial and temporal resolution requirements for IMI/ENA (Energetic Neutral Atom) and EUV (Extreme Ultraviolet) imaging instrumentation.

In this paper, the first results of a numerical study of the non-steady-state bounce-averaged kinetic equation for $\mathrm{PE}$, including their transport due to $\vec{E} \times \vec{B}$ and magnetic gradientcurvature drifts in the inner magnetosphere, are presented. The distribution function in time, spatial location in the equatorial plane, energy, and pitch angle are among the parameters calculated by the model. To insure the applicability of the bounceaveraged equations, the present study considers electrons with energies greater than $50 \mathrm{eV}$.

\section{Model}

The two main processes controlling the behavior of $\mathrm{PE}$ in the plasmasphere are their motion in the inhomogeneous geomagnetic field and Coulomb collisions with the background thermal plasma. The kinetic equation for $\mathrm{PE}$ in the guiding center approximation at altitudes greater than about $800 \mathrm{~km}$ can be written as [Khazanov et al., 1994]:

$$
\begin{gathered}
\frac{\partial f}{\partial t}+\mu \mathrm{v} \frac{\partial f}{\partial s}+\vec{V}_{D} \cdot \frac{\partial f}{\partial \vec{R}_{\perp}}+\frac{d \mathrm{v}_{\|}}{\mathrm{dt}} \frac{\partial f}{\partial \mathrm{v}_{\|}} \\
=A n_{e} \vee\left\{\frac{1}{E} \frac{\partial f}{\partial E}+\frac{1}{2 E^{2}} \frac{\partial}{\partial \mu}\left[\left(1-\mu^{2}\right) \frac{\partial f}{\partial \mu}\right]\right\}
\end{gathered}
$$

where $\left\{\mathrm{t}, \mathrm{s}, \vec{R}_{\perp}, \mu, \mathrm{v}, E\right\}$ are time, distance along a given field line, position vector in the equatorial plane, the cosine of the pitch angle, velocity, and energy, respectively; $\mathrm{v}_{\| l}=\mu \mathrm{v}$ is the velocity along the magnetic field; $\vec{V}_{D}$ is the drift velocity due to $\vec{E} \times \vec{B}$ and magnetic gradient-curvature drifts; $\vec{E}$ and $\vec{B}$ are the electric and magnetic field intensities, respectively; $f$ is the distribution function of the electrons; $\mathrm{A}=2 \pi \mathrm{e}^{4} \ln \Lambda=2.6 \times 10^{-12} \mathrm{~cm}^{2} \mathrm{eV}^{2}$ (e is the electron charge, $\ln \Lambda$ 
is the Coulomb logarithm); and $n_{e}$ is the density of the thermal plasma.

Performing the standard procedure of bounce-averaging (1) [see, for example, Lyons and Williams, 1984 or Gefan and Khazanov, 1990], the number of independent variables can be reduced to five and the kinetic equation (1) can be presented in the bounce-averaged form:

$$
\begin{aligned}
& \frac{\partial\langle f\rangle}{\partial t}+\left\langle\vec{V}_{D}\right\rangle \cdot \frac{\partial\langle f\rangle}{\partial \vec{R}_{\perp}}+\left\langle\frac{d E}{d t}\right\rangle \frac{\partial\langle f\rangle}{\partial E}+\left\langle\frac{d \mu_{0}}{d t}\right\rangle \frac{\partial\langle f\rangle}{\partial \mu_{0}}= \\
& A \vee\left\{\frac{\left\langle n_{e}\right\rangle}{E} \frac{\partial\langle f\rangle}{\partial E}+\frac{1}{2 S_{\mu} E^{2} \mu_{0}} \frac{\partial}{\partial \mu_{0}}\left[A_{\mu} \frac{1-\mu_{0}^{2}}{\mu_{0}} \frac{\partial\langle f\rangle}{\partial \mu_{0}}\right]\right\}
\end{aligned}
$$

The brackets $\langle\xi\rangle$ denote bounce-averaging:

$$
\langle\xi\rangle=\frac{1}{S_{\mu}} \oint \xi(s) \frac{d s}{\mu\left(s, \mu_{0}\right)} ; \text { where } \xi=f, \vec{v}_{D}, n_{e}
$$

and the following notation was introduced:

$$
\begin{gathered}
S_{\mu}\left(\mu_{0}\right)=\oint \frac{d s}{\mu\left(s, \mu_{0}\right)} ; \quad A_{\mu}\left(\mu_{0}\right)=\oint \frac{B_{0}}{B} n_{e}(s) \mu\left(s, \mu_{0}\right) d s \\
\quad \text { and } \mu_{0}=\frac{\mu}{|\mu|} \sqrt{1-\frac{B_{0}}{B(s)}\left(1-\mu^{2}\right)}
\end{gathered}
$$

with $B_{0}$ and $\mu_{0}$ denoting the magnetic field and the cosine of the pitch angle at the magnetic equator of the flux tube.

Note that equation (2) is not applicable to describe the PE distribution function in the loss cone. It can only be used for the description of trapped particles. The loss cone is defined by $\mu_{o b} \leq\left|\mu_{o}\right| \leq 1$ and the trapping region by $\sqrt{1-B_{0} / B(s)} \leq\left|\mu_{o}\right| \leq \mu_{o b}$, where $\mu_{o b}=\sqrt{1-B_{0} / B\left(s_{1}\right)}$ is the loss cone boundary and $B\left(s_{1}\right)$ is the magnetic field at the low altitude boundary (the region over which the electron distribution function is defined in terms of $s$ and $\mu_{0}$ is shown in Figure 1 of Khazanov et al. [1992]). That means, in order to solve equation (2), it must be coupled with a corresponding loss cone equation [Khazanov et al., 1992] or a boundary condition for the electron distribution function at the edge of the loss cone should be introduced.

Equation (2) has been transformed into the conservative form of the variables $\left(t, R_{o}, \varphi, E, \mu_{o}\right)$, where $R_{o}$ is the radial distance in the equatorial plane, and $\varphi$ is the geomagnetic east longitude, and then solved numerically in light of the ring current model of Jordanova et al. [1995], which was adjusted for the PE energy range and coupled with a field-aligned model [Khazanov and Liemohn, 1995; Liemohn and Khazanov, 1995]. Utilizing the approach developed by Fok et al. [1993], equation (2) is decomposed using a double splitting method, and advance the solution in a single dimension at each half time step. The order of the solution operators is reversed in the next half time step to achieve second order accuracy in time. The drift terms and the Coulomb drag energy term are discretized by the Lax-Wendroff scheme and the first order upwind scheme. The superbee flux-limiter method is used to switch between the high-order Lax-Wendroff scheme, which works well in smooth regions, and the low-order upwind scheme, which behaves well in the presence of strong gradients. This high resolution method gives second order accuracy on smooth solutions and well-resolved nonoscillatory discontinuities [Leveque, 1992]. The Crank-Nicholson scheme is applied for discretization of the Coulomb pitch angle diffusion term according to Khazanov [1979].
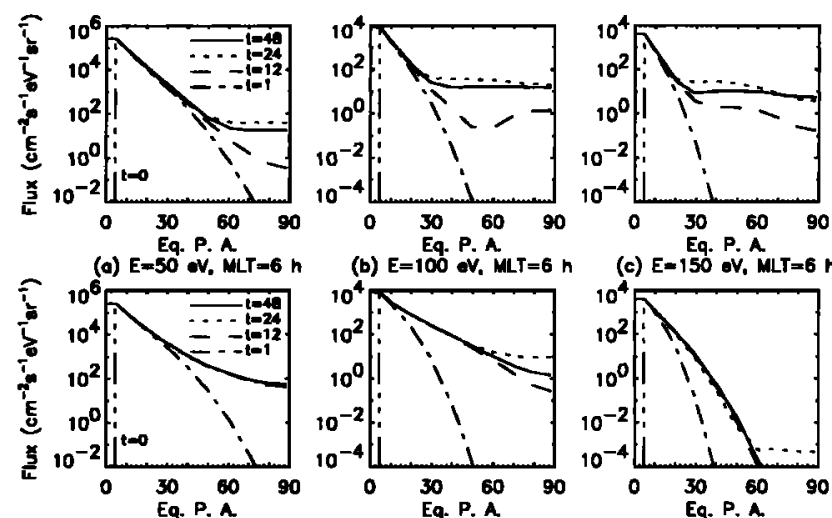

(d) E=50 $\mathrm{eV}, \hat{M L T}=12 \mathrm{~h}$

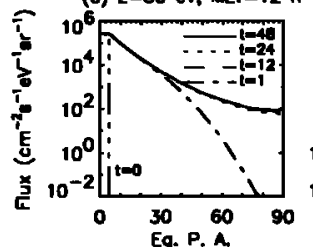

(e) E=100. P. A. $M L T=12$ h
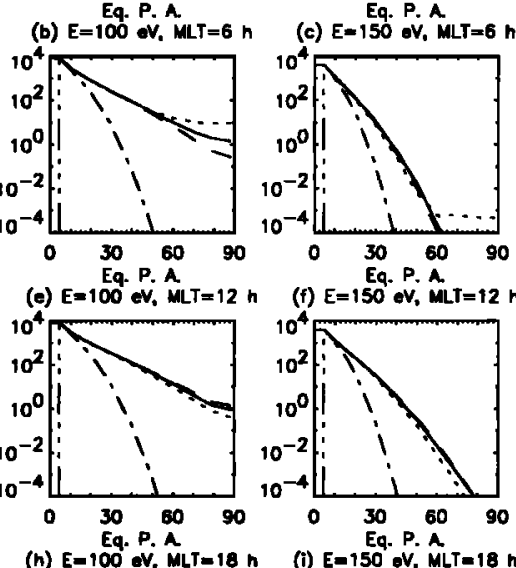

(g) $E=50$ ov, $\hat{M L T}=18 \mathrm{~h}$

(h) E=100. ‥ $\hat{\text { MLT }}=18 \mathrm{~h}$

(i) $E=150$ ov. $\hat{M L T}=18 \mathrm{~h}$

Figure 1. $L=5$ dayside pitch angle distributions.

The following boundary conditions were used: a) outside the region under consideration, $L=1.75$ to $L=6.5$, $P E$ fluxes equal zero; b) the energy range is between $50 \mathrm{eV}$ and $200 \mathrm{eV}$ with zero flux in the tail of the energy distribution; c) the solution is assumed to be periodic in azimuth $(\varphi)$; d) the only source of superthermal electrons is photoionization and secondary ionization due to these particles; and, e) the solution is assumed to be symmetric at $90^{\circ}$ pitch angle, and during the day the PE distribution function at the loss cone boundary has been calculated by using the Khazanov and Liemohn [1995] model. During the night, the absorption of PE in the ionosphere, due to the emptying of the loss cone (twice per bounce period, $\tau_{B}$ ), is taken into account by extending (2) into the loss cone and introducing here the loss term according to Lyons and Williams [1984]:

$$
\left\langle\left(\frac{\delta\langle f\rangle}{\delta t}\right)_{e}\right\rangle=-\frac{\langle f\rangle}{\tau_{e}} \text { where } \tau_{e}=\left\{\begin{array}{l}
\tau_{B} / 2 \text { in the loss cone } \\
\infty \text { out of the loss cone }
\end{array}\right.
$$

In this case it is required to use the boundary condition at zero pitch angle which is the same as in the Khazanov at al. [1994] model: $\partial\langle f\rangle / \partial \mu_{0}=\mathbf{0}$.

When considering PE formation in the plasmasphere on a global scale, it is necessary to keep in mind the possibility of PE interaction with the vast collection of plasma waves supported by the magnetospheric plasma. The wave-particle interaction mechanisms influencing the PE distribution have not been incorporated into this model yet, and as a first step will not be included in the calculation. The justification for omitting this process is as follows. The ring current and radiation belts are two major mesoscale phenomena that interact with the thermal plasma to generate most of the plasma waves in the region under consideration. Ring current-plasmasphere interaction amplifies Alfven, fast-magnetosonic, and ion-cyclotron waves, while radiation belt-plasmasphere interaction amplifies whistler mode waves. The former waves resonate with electrons at energies up to a few electron volts, and the latter 

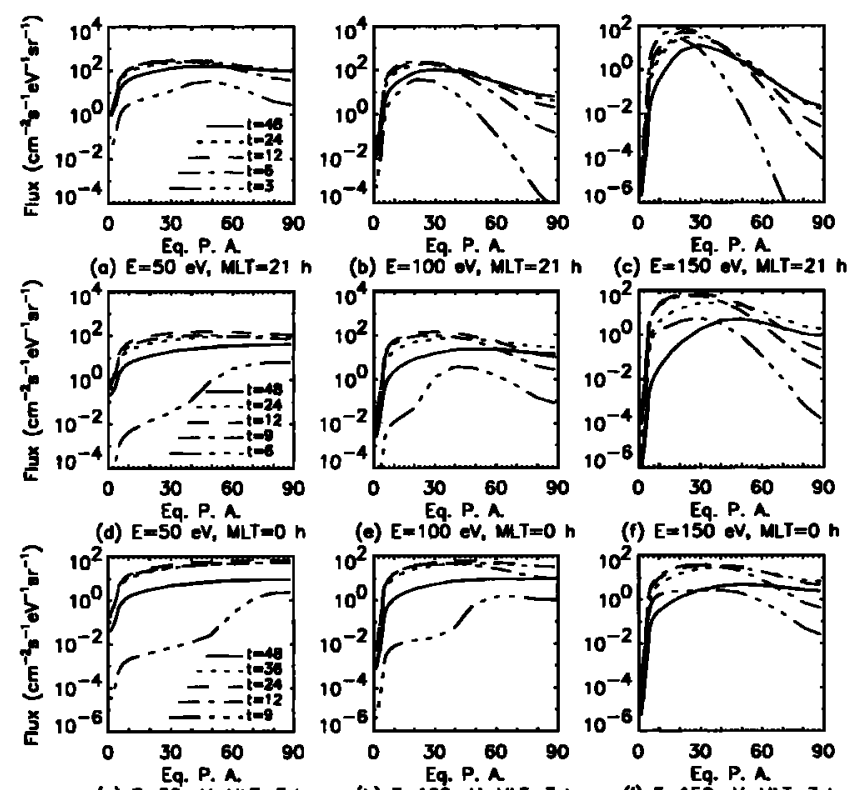

(h) $E=100$ P. $v_{1}, m T=3 n$

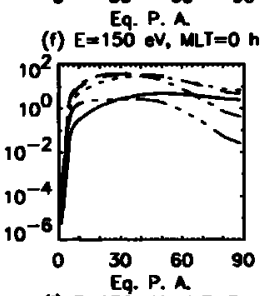

() E=150. P. $\hat{\text { QV. }}, \mathrm{MLT}=3 \mathrm{~h}$

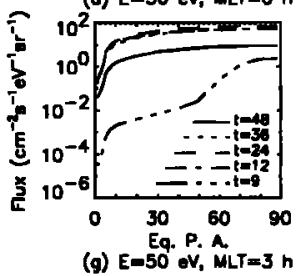

Figure 2. $\mathrm{L}=5$ nightside pitch angle distributions.

resonate at $\mathrm{keV}$ energies. Among these waves, the most appropriate candidate for additional photoelectron scattering is interaction with whistlers through Cherenkov resonance, but this will be much less efficient than Coulomb scattering for low levels of geomagnetic activity [Kuzivanov and Khazanov, 1984]. Although the energy range under consideration is usually quite stable, it degrades into the low-energy (E $\leq 50 \mathrm{eV})$ distribution, which can be unstable and generate plasma waves itself Khazanov [1979].

The following input was used to perform the PE model calculations. The bounce-averaged drift velocities of the guiding center can be obtained for the $\vec{E} \times \vec{B}$ and magnetic gradientcurvature drifts, considering that only the equatorial electric field is contributing to the bounce-averaged drift [Roederer, 1970]. The geomagnetic field in this simulation is taken to be a 3-dimensional dipole field. The electric field is expressed as the gradient of the Volland-Stern semiempirical potential model [Volland, 1973; Stern, 1975]. All of the calculations were performed for a quiet geomagnetic condition $\mathrm{K}_{\mathrm{p}}=1$ (when the main superthermal electron source is photoionization) on March 21, 1986, with F10.7 and $\langle$ F10.7 $>$ values of 150 , chosen so the atmospheric conditions are symmetric and the solar radiation is at an average intensity level. The plasmaspheric cold electrons are calculated with the model of Rasmussen et al. [1993].

Here, the first results from this new global PE model are presented and the processes of ionosphere-plasmaspheremagnetosphere PE coupling and distribution function formation on a global scale are discussed.

\section{Results and Discussion}

To describe PE formation on a global scale, the global PE transport is calculated on the time scale up to 48 hours. The early stages of the refilling process and the behavior of superthermal electrons in the geomagnetic trap on the time scale up to one hour were considered using a recently developed model [Khazanov et. al., 1993-1995; Liemohn and Khazanov,

1995], which represents a unified approach by self-consistently coupling the interaction of the two hemispheres and the capture of PE in the plasmasphere. In these papers, the readers also can find the typical PE energy and pitch angle distribution in the region of the source (below $800 \mathrm{~km}$ ) that has been used as the dayside loss cone boundary in this study. Considering PE transport based on the bounce-averaged kinetic equation (2), it is necessary to keep in mind that this approach is valid since the bounce period of $P E$ is much shorter than the scattering processes' decay lifetimes; presently just Coulomb collisions. For the time period comparable with or less than the bounce period, the kinetic equation for PE in the guiding center approximation (1) should be used, using the approach proposed by Khazanov et. al. [19931995].

In this paper, PE results above a low energy limit of $50 \mathrm{eV}$ are presented, where the applicability of the bounce-averaged kinetic equation (2) in the region under consideration $(L=1.75$ to $L=6.5$ ) is obvious. An extension of (2) to the low energy range, where the bounce period of PE is comparable with the time scales of their scattering processes, will be investigated. This would allow the inclusion of practically all important PE energies contributing to magnetospheric thermal plasma energy deposition.

It is interesting to start the calculations with initially empty flux tubes at $t=0$ on the day and night sides of magnetosphere and to consider the non-steady-state development of the photoelectron distribution function in velocity and configuration spaces as a result of photoelectron energy pumping and pitch angle redistribution in the geomagnetic trap on a global scale. The calculations in this paper have been performed only for the case of low geomagnetic activity, $K_{p}=1$; however, the model can handle any conditions of geomagnetic activity or $\mathrm{K}_{\mathrm{p}}$ history with corresponding variations in the neutral atmosphere, ionospheric and plasmaspheric densities, and electric field of magnetospheric convection.

Figure 1 shows the pitch angle distribution formation on the dayside at $\mathrm{L}=5$ for the energies $50 \mathrm{eV}, 100 \mathrm{eV}$, and $150 \mathrm{eV}$ in the different magnetic local time (MLT) sectors: MLT $=6$ is morning-sunrise (a-c), MLT $=12$ is noon (d-f), and MLT $=18$ is evening-sunset (g-i). The different lines indicate the time of the solution, which starts with the initial PE distribution located only in the loss-cone at $t=0$ and evolves to $t=48$ hours. The results of the model are shown here in terms of PE fluxes, connected with the electron distribution function according to $\phi=2 E f / m^{2}$, where $m$ is the electron mass. As seen in the presented results, in the noon-evening sector the pitch angle distribution has similar pitch angle and energy dependencies to those calculated in previous papers [Khazanov et al., 19931995]. For example, the shape of the pitch angle distributions in this time sector remains the same, it also has the same energy dependence in the transition region from the small pitch angles near the loss cone to the large pitch angles of the trap zone, and the time scale of the refilling process is proportional to $\mathrm{E}^{-3 / 2}$. In the morning, the evolution of the pitch angle distribution has a more complicated character and is similar to the dayside pitch angle distribution only during the first few hours of plasmaspheric refilling. After 10-12 hours, the shape of the pitch angle distribution in the trap zone on the morning side changes. The tail of the pitch angle distribution is trying to stretch out. There appears to be another source besides the loss cone, and the part of the pitch angle distribu- 
tion that rises depends on the PE energy. To explain such unusual pitch angle distribution behavior, attention should be given to what is going on in the nightside magnetosphere.

Figure 2 demonstrates the pitch angle distribution formation on the nightside at $L=5$ for the energies $50 \mathrm{eV}, 100 \mathrm{eV}$, and $150 \mathrm{eV}$ in different MLT sectors: MLT $=21$ is the late evening (a-c), MLT $=0$ is local midnight ( $d-f)$, and MLT $=3$ (g-i) is deep night. The different lines indicate the time of the solution, which starts with the zero PE distribution function on the nightside, and evolves to $t=48$ hours. The source of the PE on the magnetospheric nightside is the drift motion of the particles from the dayside due to the $\vec{E} \times \vec{B}$ drift in the presence of the corotation and magnetospheric convection electric fields. The main feature of the nightside PE distribution function is the sharp depletion of intensity in the loss cone, because after MLT=18, photoionization in the ionosphere disappears. PE flux in the loss cone does not go to zero, however. Depending on MLT and equatorial distance, two processes, Coulomb collisions and magnetospheric convection, move particles from the trap zone to the loss cone. These plots clearly show the time-dependent dynamics of the pitch angle development in the different MLT sectors.

As seen in Figure 2 at the deep night sector, the pitch angle distribution is flattening out at large pitch angles. This feature can be explained as a result of the conservation of the first and second adiabatic invariants as the PE drift from the dusk sector to the dawn sector along convective trajectories moving toward the Earth. Going back to the morning sector in Figure 1 (a-c), such unusual pitch angle distribution behavior in this region can now be explained simply by delivering particles from the nightside of the magnetosphere to the morning sector. Similar ideas can be proposed to explain the difference in pitch angle distribution behavior at MLT $=21$ (ac), MLT $=0$ (d-f), and MLT=3 (g-i). In this case, the late evening pitch angle distribution retains some of the shape of the dayside PE distribution.

\section{Summary}

A global PE model including transport across geomagnetic field lines due to $\vec{E} \times \vec{B}$ and magnetic gradient-curvature drifts has been developed. Using this model, a detailed calculation was performed of the PE distribution as a function of time, energy, pitch angle, and spatial location in the equatorial plane on a global scale and presented the first results of this study for a low level of geomagnetic activity.

The authors believe that the investigation of non-steadystate PE transport on a global scale is the logical next step on the path to developing a self-consistent model of the thermal plasma in the inner magnetosphere and can form the basis of a numerical space weather prediction scheme. Here, only results with photoionization and secondary production of superthermal electrons are presented, but the model is capable of handling various other sources, such as energetic precipitation and sunward flow from the magnetotail. The ultimate objective of this global superthermal electron model will be to couple it with the thermal plasma and the ring current and predict the energy deposition from superthermal and hot populations to the cold magnetospheric plasma. Extension of the energy range down to a few $\mathrm{eV}$ and up to several tens of $\mathrm{keV}$ is also planned and will provide the numerical forecast of the energy deposition from the superthermal electrons to the thermal plasma and the hot electron population on a global scale. This would allow the use of these results in cold magnetospheric plasma models to predict plasma temperatures and densities of all significant thermal plasma species on a global scale.

Acknowledgments. This work was supported at the University of Michigan by the National Science Foundation under contract ATM9412409 and by NASA under contract NGT-51335, and at the University of Alabama in Huntsville by the National Science Foundation under contract ATM-9523699. We would like to thank J. U. Kozyra and A. F. Nagy for valuable discussions on this topic. G. V. K. held a National Research Council-Marshall Space Flight Center Senior Research Associateship while this work was performed.

\section{References}

Fok, M.-C., J. U. Kozyra, A. F. Nagy, C. E. Rasmussen, and G. V. Khazanov, A decay model of equatorial ring current and the associated aeronomical consequences, J. Geophys. Res., 98, 19381, 1993.

Gefan, G. D., and G. V. Khazanov, Non-steady-state conditions of filling up the geomagnetic trap with superthermal electrons, $A n n$. Geophys. 8, 519, 1990.

Jordanova, V. K., L. M. Kistler, J. U. Kozyra, G. V. Khazanov, and A. F. Nagy, Collisional losses of ring current ions, J. Geophys. Res., in press, 1995.

Khazanov, G. V., The Kinetics of the Electron Plasma Component of the Upper Atmosphere (in Russian), Moscow, Nauka, 1979 [English translation: Washington, D. C., National Translation Center, $\# 80$ 50707, 1980].

Khazanov, G. V., and M. W. Liemohn, Non-steady-state ionosphereplasmasphere coupling of superthermal electrons, J. Geophys. Res., 100, 9669, 1995.

Khazanov, G. V., T. I. Gombosi, A. F. Nagy, and M. A. Koen, Analysis of the ionosphere-plasmasphere transport of superthermal electrons: 1. Transport in the plasmasphere, J. Geophys. Res., 97, 16887, 1992.

Khazanov, G. V., M. W. Liemohn, T. I. Gombosi, and A. F. Nagy, Nonsteady-state transport of superthermal electrons in the plasmasphere, Geophys. Res. Lett., 20, 2821, 1993.

Khazanov, G. V., T. Neubert, and G. D. Gefan, A unified theory of ionosphere-plasmasphere transport of suprathermal electrons, IEEE Transactions on Plasma Science, 22, 187, 1994.

Kuzivanov, I. I., and G. V. Khazanov, Resonance interaction of superthermal electrons with ELF radiation in the Earth's plasmasphere, Geomagnetism and Aeronomy, 24, 485, 1984.

Leveque, R. J., Numerical Methods for Conservation Laws, 2nd ed., Birkhäuser Verlag, Boston Mass., 1992.

Liemohn, M. W., and G. V. Khazanov, Non-steady-state coupling processes in superthermal electron transport, Coupling of Micro- and Mesoscale Processes in Space Plasma Transport, in press, 1995.

Lyons, L. R., and D. J. Williams, Quantitative Aspects of Magnetospheric Physics, D. Reidel Publishing Company, Boston Mass., 1984.

Rasmussen, C. E., S. M. Guiter, and S. G. Thomas, Two-dimensional model of the plasmasphere: refilling time constants, Planet. Space Sci., 41, 35, 1993.

Roederer, J. G., Dynamics of Geomagnetically Trapped Radiation, Springer-Verlag, New York, 1970.

Stern, D. P., The motion of a proton in the equatorial magnetosphere, $J$. Geophys. Res., 80, 595, 1975.

Volland, H., A semiempirical model of large-scale magnetospheric electric fields, J. Geophys. Res., 78, 171, 1973.

G. V. Khazanov, T. E. Moore, M. W. Liemohn, M.-C. Fok, Space Sciences Laboratory, NASA/MSFC, ES-83, Huntsville, AL 35812 (khazanov@mpb.msfc.nasa.gov).

V. K. Jordanova, Department of Atmospheric, Oceanic, and Space Sciences, University of Michigan, Ann Arbor, MI 48109.

Received: July 19, 1995; revised: November 14, 1995; accepted: December 29, 1995. 